2017-08-01

\title{
Survivability of wave energy converters using CFD
}

Ransley, E::0000-0003-1446-7907

http://hdl.handle.net/10026.1/8587

10.1016/j.renene.2017.03.003

Renewable Energy

Elsevier BV

All content in PEARL is protected by copyright law. Author manuscripts are made available in accordance with publisher policies. Please cite only the published version using the details provided on the item record or document. In the absence of an open licence (e.g. Creative Commons), permissions for further reuse of content should be sought from the publisher or author. 


\title{
Survivability of Wave Energy Converters using CFD
}

\author{
E. J. Ransleya,*, D. Greaves ${ }^{\mathrm{a}}$, A. Raby ${ }^{\mathrm{a}}$, D. Simmonds ${ }^{\mathrm{a}}$, M. Hann ${ }^{\mathrm{a}}$ \\ ${ }^{a}$ School of Marine Science and Engineering, Plymouth University, UK
}

\begin{abstract}
The survivability of Wave Energy Converters (WECs) in extreme waves is a critical issue faced by developers; typically assessed via small scale physical experiments with considerable uncertainties. Until recently, confidence in the ability of numerical tools to reproduce extreme wave events and their interaction with floating structures has been insufficient to warrant their use in routine design processes. In this work a fully nonlinear, coupled tool for simulating focused wave impacts on generic WEC hull forms is described and compared with physical measurements. Two separate design waves, based on the 100 year wave at Wave Hub and using the NewWave formulation, have been reproduced numerically as have experiments in which a fixed truncated circular cylinder and a floating hemispherical-bottomed buoy are subject to these focused wave events. The numerical model is shown to reproduce the wave events well with some inaccuracies observed in shallower water depths. The results for pressure and run-up on the cylinder surface, as well as the high-order free-surface behaviour, also compare well with experimental results. The floating buoy's motion and mooring load are reproduced exceptionally well showing that the tool presented here can be used to assess WEC survivability and complement existing physical modelling.
\end{abstract}

Keywords: NewWave, truncated cylinder, floating buoy, 6DOF motion, mooring, OpenFOAM

2015 MSC: 00-01, 99-00

${ }^{*}$ Corresponding author
Email address: edward.ransley@plymouth.ac.uk (E. J. Ransley)

Preprint submitted to Renewable Energy

January 30, 2017 


\section{Introduction}

In order to reduce the Levelised Cost of Energy (LCOE) of wave energy, research directly concerning Wave Energy Converters (WECs) has focused primarily on optimising the response of devices [1, 2]; the majority of formal design 5 guidance concentrates on the operational conditions of the device [3]. However, this is not always the most critical factor in a design context. Defined by the UK Energy Research Centre [4] as 'the ability to survive predicted and surprise extremes in wind, wave and tidal current conditions, in any combination', survivability has now been identified as a key issue for the marine renewables industry, posing a significant challenge requiring complementary development and underpinning research. Crucial to both the economic and environmental success of a WEC [5], it is widely accepted that the current design procedures for the operational envelope must be complemented with a second level of design which considers the 'survival envelope' [6, 7]. Long-term reliability and failure rate estimates for WEC components have been attempted, but a lack of available data makes such analysis difficult [8]. Furthermore, few short-term survivability assessments of complete $\mathrm{WEC}$ and mooring systems have been completed. Wave impact, green water and the episodic mooring loads experienced by WEC systems have received very little attention, with the exception of the conceptual criteria proposed by some developers who prioritise survival over power capture ensuring devices feature inherent load shedding and de-tuning capabilities in large waves $[9$.

In terms of short-term survivability, it is the sometimes catastrophic impacts from abnormally large, 'freak' or 'rogue', waves which are of most concern to WEC developers. However, current guidelines on the design and operation of WECs fail to provide definitive guidance on how to prepare dynamic, coupled $\mathrm{WEC}$ and mooring systems for these discrete events [3, 5]. These events have typically been reproduced via long-term, time domain simulations of extreme, irregular sea states restricting their analysis to physical experiments or 
numerical models with low CPU demand [10. Deterministic representations of extreme waves have started to emerge [11. In particular, the 'design-wave' known as 'NewWave' is referred to extensively in offshore engineering and has become the industry standard for modelling extreme wave interactions with offshore structures [12, 13, 14]. Despite this, the specific wave group combinations 35 responsible for the most severe loads on dynamic structures remain unclear and can, currently, only be found by testing a range of conditions [10, 15. Uncertainty over WEC survivability, caused by a lack of rigorous design guidelines, a poor understanding of device behaviour in extreme waves and ambiguity over the precise conditions responsible for the maximum load cases, represents an insurmountable risk to potential investors and contributes significantly to the industry's stagnation [4, 16. If wave energy is to become an affordable part of the energy mix, potential design solutions require a greater understanding of the hydrodynamics and structural loading experienced during extreme events in order to mitigate the risk of device and mooring failure.

45 The continued increase in the performance-to-cost ratio of modern computers has meant that numerical models can now provide the quantitative description required for engineering analysis as well as a means to interpret the fundamental phenomenological aspects of experimental conditions at full-scale that physical tests may not [17]. Consequently, the guidelines on design and 50 technology readiness of WECs state that optimisation of both the vessel and mooring system should typically now be performed on a computer [18, 3, 19. In cases with highly nonlinear local effects or strong nonlinear interactions between waves and structures, however, there is a lack of confidence in the predictive capability of numerical tools to provide extreme motions and loads on the hull, 55 Power Take-Off (PTO), moorings and anchors. In addition to this, numerical tools capable of including the required full range of physics tend to have excessive execution times making their use in routine design applications prohibitive. Survival tests are, therefore, considered to be a specialised experimental condition and physical tests are still preferred over numerical models when assessing ${ }_{60}$ the seaworthiness of a WEC [3, 19]. Despite this, there remain a number of 
uncertainties surrounding extreme wave loading on full-scale WECs as wave tank survival testing can typically only be performed at small scales $(\approx 1: 50)$ [17] when larger scales are highly recommended [19, 10. Therefore, if both the confidence in and the efficiency of fully nonlinear coupled numerical models could be increased, the design and survivability of full-scale WECs could be significantly enhanced.

The aim of this work is, therefore, to develop a computational tool which can provide a reliable understanding of the behaviour of WEC systems under pxtreme wave loading. In this article, a fully nonlinear Computational Fluid Dynamics (CFD) approach has been used to model the free surface interaction of focused waves with generic WEChull forms. A systematic, incremental development procedure has been implemented and validated against experimental data to assess the predictive capability of the tool at each stage of increasing complexity. Initially, the propagation of two different dispersively focused NewWaves are considered, then a fixed truncated surface-piercing cylinder is introduced before the interaction of a focused wave with a floating hemispherical-bottomed buoy with a linear spring mooring is simulated.

\section{The Numerical Wave Tank (NWT)}

The numerical simulations described in this article are performed within a Numerical Wave Tank (NWT) based on the work of Ransley 20]. The method utilises the open-source CFD libraries OpenFOAM ${ }^{\circledR}$ to solve the ReynoldsAveraged Navier-Stokes (RANS) equations for two incompressible, isothermal, immiscible fluids using a Volume Of Fluid (VOF) based interface capturing scheme [21, 22, 23. Wave generation and absorption is achieved using the expression-based boundary conditions and passive Relaxation Zone formulation included in the additional toolbox waves2Foam [24, 25]. An exponential decay function is used as the relaxation weighting factor throughout this study.

In the floating buoy case, a Six Degrees Of Freedom (6DOF) rigid body motion solver is used to simulate the coupled motion of the body. This solver 
is distributed with OpenFOAM ${ }^{\circledR}$ and allows for automatic mesh motion in a portion of the domain (described by a radial distance parameter) via a moving wall velocity condition and a force calculation on the patch describing the boundary of the body. The position of grid nodes coinciding with the surface of the structure are then updated accordingly at every timestep as is the rest of the mesh based on spherical linear interpolation with a cosine profile in the distancing function [26].

\section{Design waves (NewWave)}

It is now widely accepted that present design procedures for the operational envelope need to be complemented by a second level of design which takes into

Fortunately, the idea of an extreme wave allows for the notion of a single event with a specific shape, and crest height, $\eta_{c r}$, over a single associated period. 
Then a convenient 'design-wave' can be constructed to examine the peak surface elevations and loads due to extreme events in a reproducible, deterministic way. In offshore engineering a design-wave known as 'NewWave' is referred to extensively and has become the industry standard for modelling extreme wave interactions with offshore structures [12, 13, 14]. NewWave relies on the dispersive nature of water waves to produce an extreme wave event at a specific point in space and time by combining smaller, sinusoidal components of different frequencies. Retaining the broad-banded nature of extreme ocean waves, the linear NewWave has a shape based on the average extreme in a linear, random, Gaussian sea and is proportional to the auto-correlation function (the Fourier transform of the sea state power spectrum in question) [12. By discretising this definition into a finite number of sinusoidal components, $N$, and limiting ourselves to uni-directional seas, a linear, crest-focused wave group then has the surface elevation

$$
\eta(x, t)=\sum_{n=0}^{N} a_{n} \cos \left(k_{n}\left(x-x_{f}\right)-\omega_{n}\left(t-t_{f}\right)\right),
$$

where $x_{f}$ and $t_{f}$ are the target position and target time respectively. For a linear NewWave the individual component amplitudes are given by

$$
a_{n}=\frac{A_{c r} S_{n}(\omega) \Delta \omega_{n}}{\sum_{n} S_{n}(\omega) \Delta \omega_{n}}
$$

where $S_{n}(\omega)$ is the energy spectrum, $\Delta \omega_{n}$ is the frequency increment and $A_{c r}$ is the linear crest amplitude given by

$$
A_{c r}=\sqrt{2 m_{0} \ln (N)}
$$

where $m_{0}$ is the zeroth moment of the spectrum. Equation 3 then ensures a NewWave model of the largest wave in $N$ waves. It is generally accepted that a three hour sea state has approximately 1000 waves and so $N=1000$ [30].

NewWave has been shown to model the underlying linear part of large ocean waves in both deep and intermediate water depths [31, 32. It has been shown to outperform traditional 5th order Stokes wave methods when predicting the forces on offshore space-frame structures [29]. However, there still exists some 
concern over the use of NewWave in shallow water as dispersion has less effect and nonlinearities become significantly more important [33, 34]. Higher-order corrections to the NewWave definition have been proposed [12] and the subject remains a source of ongoing research. Despite this and due to limitations with the physical wave maker software used to generate the validation data for this work, a linear NewWave approximation will be used to describe the incident extreme waves. Any nonlinearities present at the position of the wave maker will be ignored. Furthermore, only unidirectional waves will be considered in this work. It is believed that, even with these simplifications, the use of a NewWave profile provides a more probable version of an extreme wave compared to those used in similar applications which tend to use either 5th order Stokes waves or focused waves based on generic ocean spectra (e.g. Pierson-Moskowitz (PM) or Joint North Sea Wave Observation Project (JONSWAP)] [35, 36]. In order to limit the effect of this linearised input signal it can be argued that the position and time of the extreme event should be such that the maximum crest amplitude (and so contribution of nonlinearities) at the wave maker is minimised. Bateman et al. 13] discuss how this leads to unfeasibly long simulations but provided the second-order corrections are within $4 \%$ a relatively short simulation is sufficient to allow the nonlinearities to form after generation. It is therefore believed that, positioning the target location sufficiently far from the wave maker allows suitable time and distance for nonlinearities to form and thus produce a realistic, fully-nonlinear, extreme wave result. Further-still, it has been shown that global loads calculated using NewWave with second-order corrections are the same as those derived using the equivalent linear NewWave [29. Therefore, it is hoped that this simplification will not reduce the applicability of the results produced here when compared to realistic extreme ocean waves.

\subsection{Validation experiments - NewWave}

The controlled generation of waves for the purpose of experimentation, either physical or numerical, requires the specification of some driving mechanism (wave paddle motion or inlet boundary condition) typically based on a theoret- 
ical description of the desired wave shape and kinematics. There are a number of wave generation methods possible in NWTs, and in this work an expressionbased, inlet boundary condition is applied, allowing for wave generation based on a set of generic wave theories [24. These theories specify the time evolution of the surface elevation, and hence the volume fraction, as well as the vector velocity at each point on the inlet boundary. The mixture pressure $(p-\rho g h)$ on the inlet is then given a zero gradient boundary condition.

As the aim of this project is 'to provide a reliable understanding of the behaviour of WEC systems', the applications investigated here are all designed to reproduce a scaled physical experiment. This provides a measure of the validity of the NWT developed by allowing comparisons between the numerical results and those produced in a controlled environment using a real fluid. Only then can this tool be used to explore cases beyond the capabilities of physical experiments, such as those at full scale, with any confidence in the results.

All of the experiments used for validation purposes in this article have been performed in the Ocean Basin at Plymouth University's Coastal, Ocean And Sediment Transport (COAST) laboratory. Unidirectional NewWaves are physically generated by 24 flap-type wave paddles at one end of the $35 \mathrm{~m}$ long and $15.5 \mathrm{~m}$ wide wave tank. The depth is $4 \mathrm{~m}$ at the wave paddles (which have a hinge depth of $2 \mathrm{~m}$ ) and decreases linearly to a depth which can be varied between 0 and $3 \mathrm{~m}$ (utlilising a raisable floor). There is a parabolic beach at the far end of the basin, opposite the wave makers.

A NewWave profile is produced, at a wave gauge located some distance from the wave makers, by combining 243 wave components with frequencies uniformly spaced between 0 and $2 \mathrm{~Hz}$ and amplitudes generated using NewWave theory. The phases of the wave components are found using a 'trial-and-error' adjustment that accounts for the nonlinear interactions present in the wave propagation and ensures a symmetrical waveform at the point of interest, i.e. a focused wave [37, 38, 15. Unfortunately this does not guarantee the precise NewWave is produced and leaves a number of unanswered questions regarding the kinematics of the wave at the target location. 
In an attempt to remove the complexities of the fluid flow in the vicinity of the wave paddles, improve the computational efficiency of simulations and eliminate any error in the predictions made by the wave maker software, the method used in this work employs an additional wave gauge situated $8 \mathrm{~m}$ from the wave maker - the 'forward' wave gauge. This then serves as the numerical input data for the simulation. It is hoped that any effect due to the wave generation or the depth change near the wave maker will have occurred by the time the wave reaches this wave gauge, from where a constant depth can be assumed. The drawback of this method is that only the surface-elevation is recorded at the forward position. The velocity is also needed to fully define the inlet boundary conditions. Therefore, the time series recorded at the forward wave gauge is reduced into linear wave components using a Fast Fourier Transform (FFT) The velocity is then approximated as the linear sum of velocities arising from the linear velocity potential for each component.

\subsection{NewWave case 1}

The first design wave considered is based on the 100 year conditions at the Wave Hub, a designated test area for pre-commercial wave energy devices located $16 \mathrm{~km}$ off the north coast of Cornwall, UK [39. A Weibull fit was used to approximate the frequency spectrum for the 1 in 100 year sea state at the site. Then a NewWave, based on the JONSWAP spectrum $\left(H_{s}=14.4 \mathrm{~m}\right.$ and $\left.T_{z}=14.1 s\right)$ was produced experimentally at a scale of 1:30 with the depth correctly scaled to the $52 \mathrm{~m}$ deep Wave Hub site, i.e. $1.73 \mathrm{~m}$.

This wave was reproduced numerically in a $25 \mathrm{~m}$ long and $1.73 \mathrm{~m}$ deep, $2 \mathrm{D}$ computational domain with a $13 \mathrm{~m}$ long relaxation zone installed at the end opposite the inlet boundary. The mesh was composed of square cells and had a resolution, in the region containing the free surface, of 36 cells over the maximum wave height $(\approx 0.6 \mathrm{~m})$. Based on the work by Ransley [20, this was deemed sufficient to ensure mesh independence with a Root Mean Squared (RMS) error within $2 \%$. In order to improve the simulation efficiency, the rest of the domain was set two levels of refinement less with 3 layers of cells between refinement 

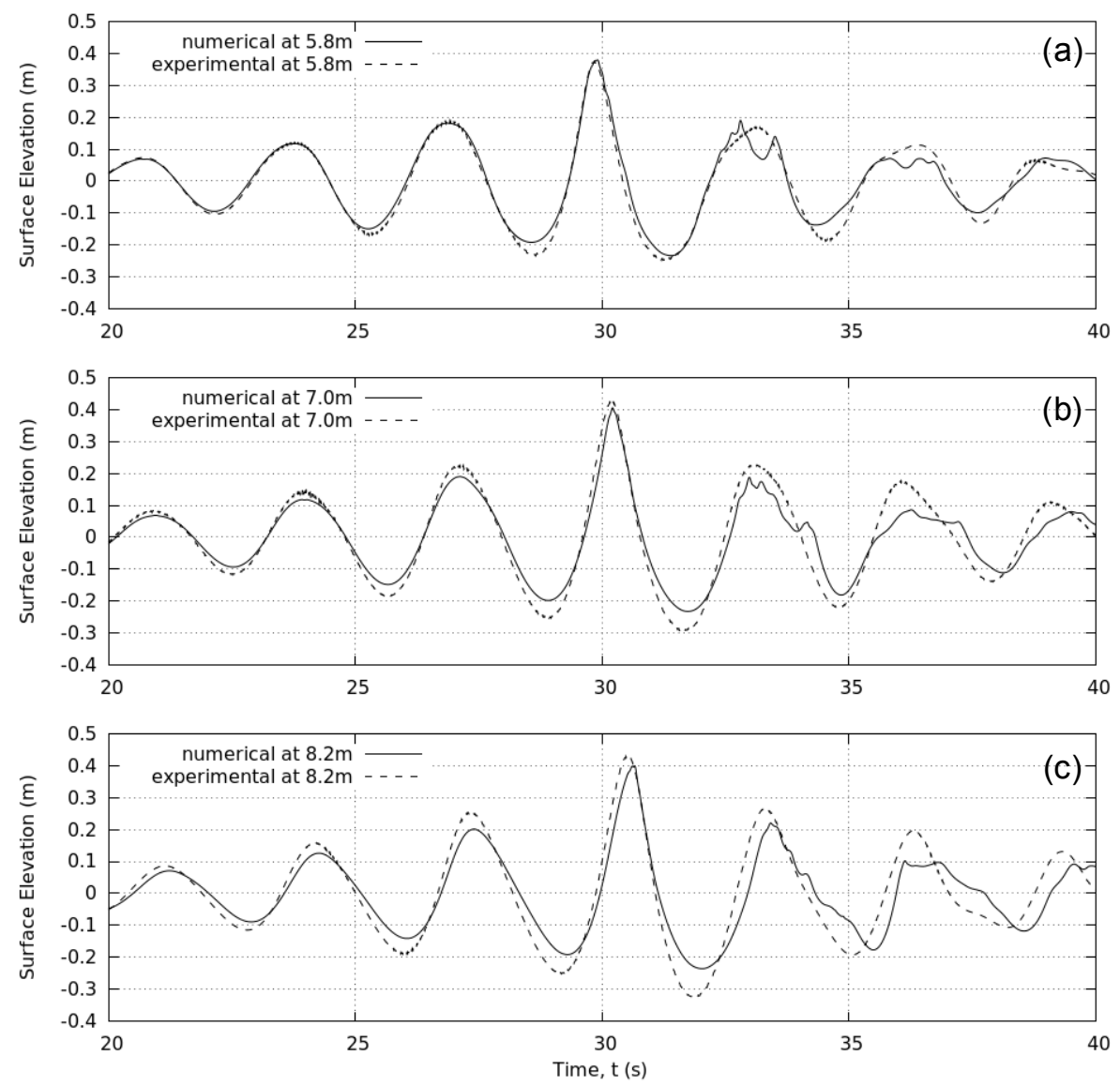

Figure 1: Comparisons between the experimental and numerical surface elevation of a NewWave with a target at $5.8 \mathrm{~m}$ measured at three separate places: the target position $(5.8 \mathrm{~m})$ (a), $7.0 \mathrm{~m} \mathrm{(b)} \mathrm{and} 8.2 \mathrm{~m}$ (c).

levels. A mesh dependency study shows no noticeable difference in the surface elevation at the target location when using this mesh instead of a uniform mesh, i.e. the simulation is still mesh independent. Furthermore, no difference was observed when the Courant-Friedrichs-Lewy (CFL) condition was relaxed to have the maximum Courant number for the phase fields (maxAlphaCo) and the other fields (maxCo) equal to 0.5 instead of 0.25 . Therefore the former has been used in this case. 
The resultant free surface elevation at the target location $(5.8 \mathrm{~m}$ from the inlet boundary/forward wave gauge position) is shown in Figure 1 1 along with that from the physical experiment. The main crest in the numerical simulation is reproduced well, however, there are a number of discrepancies. The troughs are noticeably shallower in the numerical result and the surface elevation has an irregular form after the main crest, which does not resemble reflected waves from the end of the NWT. It has been noticed that, for steeper waves and shallower water depths, the numerical wave can become unstable at the trailing edge of the main crest resulting in behaviour similar to localised wave breaking. The current explanation for this is that the linear approximation for the velocities at the inlet boundary is a poor representation of those actually present in the physical experiment and this is particularly problematic when the main crest of a steep wave is generated in shallow water. Furthermore, it is insufficient to judge the quality of the numerical solution based on a single surface elevation time series. Even a perfect recreation of the surface elevation at a single point does not guarantee the other flow parameters, such as velocity, are also matched. At the very least, if the solution is accurate, the comparison of surface elevation time series at multiple locations should be equally good. This would suggest the propagation of the wave, and hence the solution for the fluid velocities, was adequate and provides greater confidence in the numerical result. Therefore, Figure $1 \mathrm{p}$ and c show the solution further down the tank. As can be seen, the comparison worsens as the wave propagates down the basin. It is possible that a difference in the reflections in both the physical and numerical tests are to blame. However, it is again suspected that the velocities specified on the numerical inlet boundary do not perfectly match those in the experiment leading to variations in the propagation of the wave throughout the wave tank.

\subsection{NewWave case 2}

In a second design wave case, another NewWave based on the 100 year event at the Wave Hub site was produced. The experiment, in this case, was performed at 1:50 scale and a $\mathrm{PM}$ spectrum with an $f_{p}$ of $0.356 \mathrm{~Hz}$ was used 
instead of a JONSWAP (as in the previous case). The result is a more compact wave packet. In contrast to the first case, the depth was set at $2.8 \mathrm{~m}$, reducing the severity of the depth change in front of the paddles. It was hoped that this case, with a reduced depth change, would provide a test for the NWT without the inlet issues discussed above. As a consequence the depth was not correctly scaled to the Wave Hub site, the conditions in this case therefore represent a deep water $(140 \mathrm{~m})$ site instead of an intermediate one such as Wave Hub.

Another two-dimensional NWT was created and the experimental waves were reproduced numerically using the same forward wave gauge method as above. The numerical domain was $18 \mathrm{~m}$ in length with an $11 \mathrm{~m}$ relaxation zone on the outlet. In this case, the mesh was again made of square cells this time with a side length of $0.037 \mathrm{~m}$ in the region containing the free surface, the rest of the mesh was one level of refinement less. Based on the convergence work of Ransley [20], this was again deemed sufficient for an RMS error of within $2 \%$ based on a wave steepness $(H / \lambda)$ of 0.04 . Again, maxCo and maxAlphaCo were set at 0.5 .

Comparisons between the experimental and numerical results for the surface elevation at a number of distances from the inlet are shown in Figure 2, As can be seen, the NWT has performed exceptionally well in this case. The main trough in Figure $2 a$ is within $2 \%$ of the experimental value and the main crest in 2 is within $6.5 \%$. Furthermore, unlike in the previous case, the comparative surface elevation results are equally good across a significant distance suggesting that the full range of flow variables have been captured correctly. Perhaps in this case the reduced scale, more compact wave packet and increased depth mean that linear superposition provides a better approximation to the actual wave than in the first case.

These results provide confidence in the ability of the NWT to reproduce physical experiments of extreme waves. The forward wave gauge method and the approximation of linear superposition, for specifying the numerical inlet boundary conditions, appear to be sensitive to both the water depth and the nonlinearity of the wave being generated. It would appear that steep, shallower 

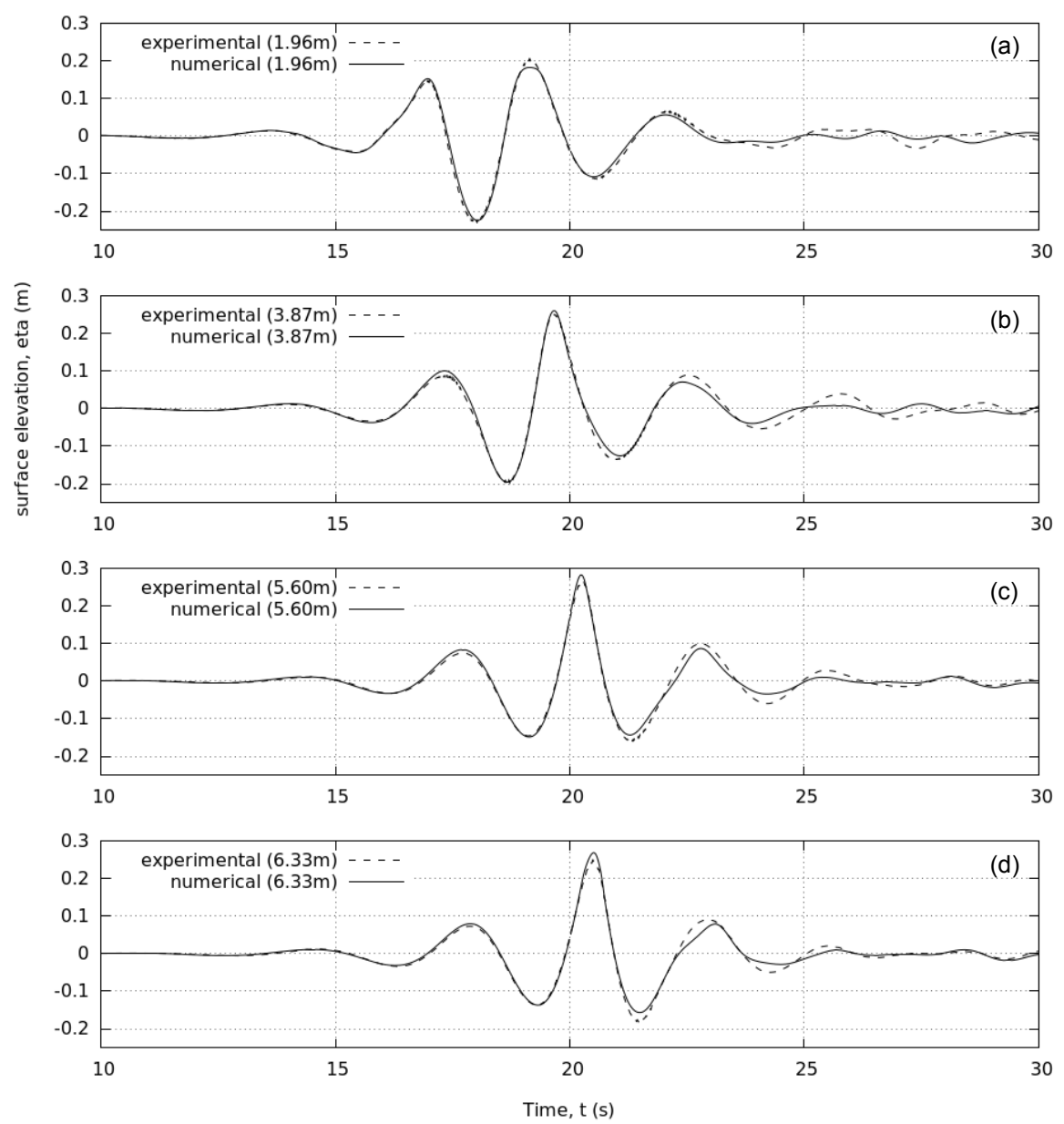

Figure 2: Comparison between the experimental (grey) and numerical (black) surface elevation of the second focused wave case measured at four separate distances relative to the numerical inlet: (a) $1.96 \mathrm{~m}$, (b) $3.87 \mathrm{~m}$, (c) $5.6 \mathrm{~m}$ and (d) $6.33 \mathrm{~m}$.

water cases require an improved definition of the flow properties at the forward wave gauge position (utilising higher-order wave theories). 


\section{Fixed Truncated Circular Cylinder}

Cylinders form the key structural components of many offshore structures and are also often assumed to be a good approximation to many more complicated geometries. For example, bottom-mounted, surface-piercing cylinders have been used to support causeways and piers as well as more recent developments like offshore wind turbines. Truncated cylinders, beyond simple marker buoys, are used for buoyancy in a range of floating structures including semisubmersibles, spar buoys and Tension-Leg Platforms (TLPs) as well as floating offshore wind applications [0]. Furthermore, the elements of space-frame structures, sub-sea umbilicals, risers and flowlines all tend to be cylinders. As waves with cylinders, ranging from analytical solutions which take advantage of the simple geometry to fully nonlinear work concerning the fundamentals of more complicated flow structures. It is for this reason that a surface-piecing truncated circular cylinder has been chosen as a validation case here; not only

In 1950 Morison et al. 41 published their influential work on the force exerted by surface waves on piles. Modified versions of the Morison equation, including the effects of turbulence and vortices, are still widely used in applications involving the interaction of non-breaking waves with offshore structures,

Furthermore, empirical methods cannot be valid for all flow regimes and scaling results can be problematic.

It was not until much later that nonlinear, interface-resolving models were developed and the hydrodynamics, run-up, and surface elevation in the vicinity 

impulsive force coming from a breaker front, or tongue. Typically the force from breaking wave impacts has been calculated as the sum of a quasi-static component, derived using the Morison equation, and a dynamic component involving an empirical measure of the impact area known as the curling factor, ${ }_{345} \lambda_{c}$ [4, 46]. More recently, fully nonlinear CFD models of breaking wave im- 
pacts on cylinders have been published and compared with estimates from the Morison equation. These have shown that the simpler Morison-type approach is capable of predicting the general trends during inertia dominated cases but not extreme wave loading [47, 35]. Despite this, breaking wave impacts will not be considered in this work as crucial phenomena such as fluid aeration and entrapment of air pockets are not accounted for in the incompressible solver used here. Nevertheless, the evidence in the literature suggests that considerable nonlinear effects, crucial for the assessment of survivability, occur during the interaction of steep waves with fixed cylinders and that simpler numerical models do not reproduce these accurately.

\subsection{Validation experiment - Cylinder}

To provide validation data for extreme wave loading on fixed, WEC like structures, a physical experiment was conducted in which a fixed, vertical, truncated cylinder was subjected to the same 100-year wave as that from NewWave case 1 above. In this case the location of maximum surface elevation was approximately coincident with the position of the cylinder's axis and the water depth was again $1.73 \mathrm{~m}$ in order to represent the Wave Hub site at 1:30 scale. The cylinder's axis was positioned $8.2 \mathrm{~m}$ from the forward wave gauge position and had a diameter of $0.4 \mathrm{~m}$ and a draft of $0.4 \mathrm{~m}$ to represent a point-absorbertype WEC with a diameter and draft of $12 \mathrm{~m}$. The run-up and pressure on the front of the cylinder were measured using a wave gauge mounted in front of the cylinder and a flush, diaphragm, pressure transducer fitted at the Still Water Level (SWL) respectively. The physical experiment was repeated 3 times and it was found that there was no noticeable difference between the results in each case for either the run up or the pressure measurements.

\subsection{Numerical setup - Cylinder}

To reproduce this case numerically, a $25 \mathrm{~m}$ long and $6 \mathrm{~m}$ wide computational domain was created. The water depth was $1.73 \mathrm{~m}$ and the air phase was given a height of $1 \mathrm{~m}$. The computational mesh was made primarily of cubic cells. The 

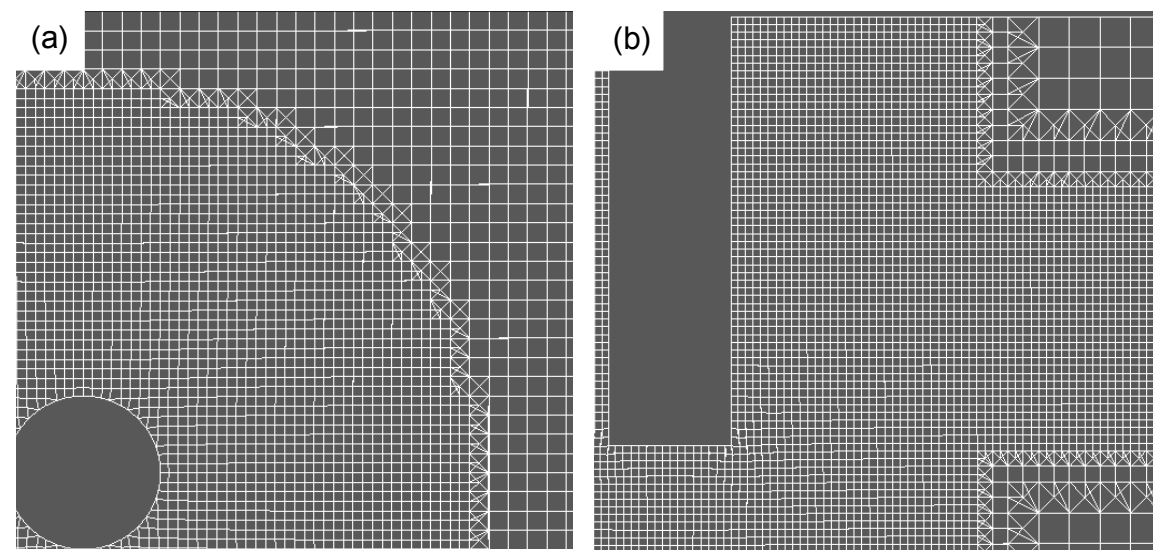

Figure 3: Part of the mesh used in the fixed cylinder cases: (a) plan view (slice $0.5 \mathrm{~m}$ above SWL), (b) cross-section of region containing the cylinder (slice along centre-line).

region containing the free surface had a cell side length of $0.025 \mathrm{~m}$ and the rest of the mesh was two levels of refinement less with a minimum of three cells between levels. In addition to this, a cylindrical region with a radius of $1 \mathrm{~m}$, stretching the full height of the domain and sharing an axis with the cylinder, was given the same resolution as the free surface. The region occupied by the cylinder, centred $8.2 \mathrm{~m}$ from the inlet boundary, was removed from the domain using the snappyHexMesh utility which offers a wide variety of options and quality control measures to ensure the mesh quality is not degraded significantly by the addition of complex boundary geometries. Figure 3 shows the mesh in the vicinity of the cylinder. As in the NewWave case 1, a $13 \mathrm{~m}$ relaxation zone was applied to the end of the NWT. A $1 \mathrm{~m}$ relaxation zone was positioned on the inlet to absorb any reflected waves from the cylinder which was given no-slip wall-type boundary conditions (as were the front and back boundaries of the domain).

\subsection{Results - Cylinder}

390 The run-up and pressure results, compared with corresponding physical results, are shown in Figures 4 and 5 respectively. The run-up time series is 


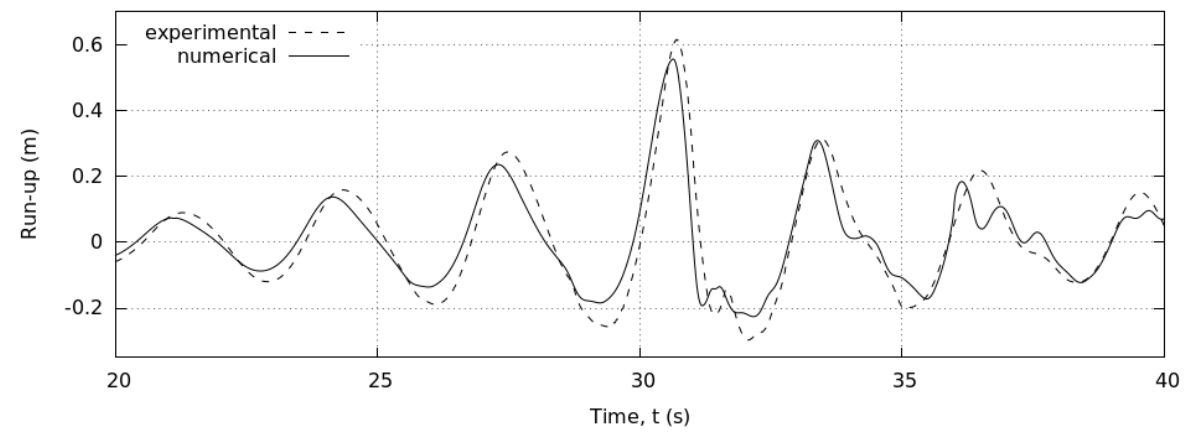

Figure 4: Run-up on the front of a cylinder. Numerical (black); physical (grey).

very similar to that of the wave-only case (NewWave case 1 above), but with increased amplitude and an additional small peak immediately after the main crest; the pressure has a series of peaks which coincide with the peaks in run-up whilst troughs appear as zero pressure due to the transducer drying. There is a consistent anomaly at the beginning of each of the pressure records which has been attributed to 'thermal shock' (the effect of temperature changes on the transducer electronics). Once the transducer is fully submerged, i.e. when the first waves arrive, this effect is resolved and the anomaly is removed. Finally, some of the early smaller pressure peaks predicted by the numerical simulation, in Figure 5 are not observed in the physical data. This is because the $1 \mathrm{bar}$ $(100 \mathrm{kPa})$ pressure transducer is operating at the limit of its sensitivity in this case and the measurement is at the same level as the noise in the signal.

Considering the differences between the physical and numerical results in the wave-only case (Figure 1), the numerical simulation provides a good approximation of the run-up and pressure on the front face of the cylinder. The run-up comparison is particularly good during the low amplitude part of the wave packet. Between 20 and $40 \mathrm{~s}$ the run-up shows the same pattern of discrepancies as the surface elevation results in the absence of the cylinder (Figure ${ }_{410}$ 1 ;). It is therefore likely that the run-up would be predicted accurately if the reproduction of the wave-only case was improved. 


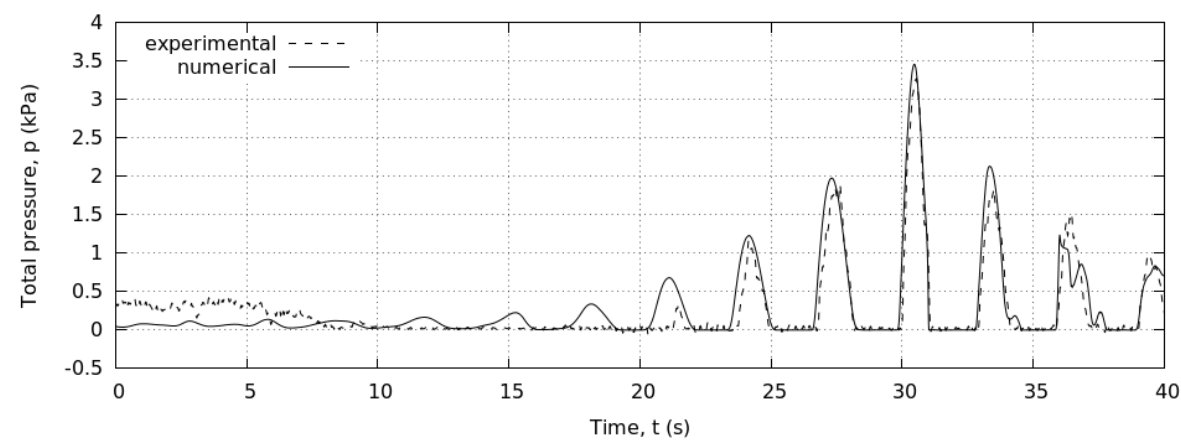

Figure 5: Pressure on the front of a cylinder, at the SWL Numerical (black); physical (grey).

One element worth further investigation is the additional peak on the trailing edge of the main crest. From observations made during the physical experiments a few interesting phenomena have been noted. Until the first of the three main crests, the water surface is undisturbed, no scattered waves are observed and the run-up is both comparable in amplitude and phase to the incident wave. As the larger crests arrive the difference in run-up around the cylinder's circumference increases significantly and some symmetrical wave scattering is observed in the lee of the cylinder. A deep trough is formed to the rear as run-up considerably greater than the incident wave height is produced on the front of the cylinder. There is a thickening of the leading edge of the run-up jet when the wave crest is alongside the cylinder. There is then an upwelling on the back of the cylinder with pronounced depressions on either side. The free surface then collapses backwards at the rear of the cylinder producing a spilling broken wave which propagates upstream causing a dip and then a small additional peak in run-up on the front of the cylinder as it passes. Figure 6 shows snapshots at the point of maximum run-up (top) and $0.4 \mathrm{~s}$ later (bottom) during the physical experiments (left) and the numerical simulation (right). It can be seen that, qualitatively, the numerical solution reproduces the physical phenomena associated with run-up of a NewWave on a fixed truncated cylinder well. Despite the use of a truncated cylinder, the results from this investigation are very similar to the observations 

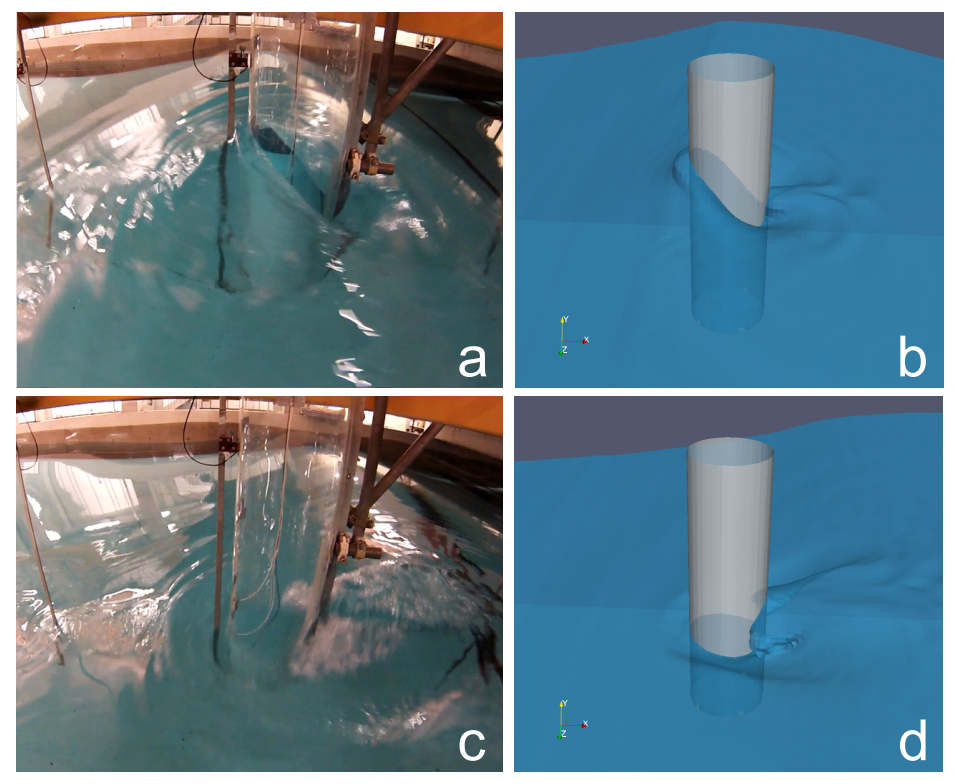

Figure 6: Snapshots at maximum run-up (top) and $0.4 \mathrm{~s}$ later (bottom) during the physical experiment (left) and the numerical simulation (right).

of Chaplin et al. 48, when assessing the secondary loading cycle and ringing of a vertical cylinder in steep non-breaking waves. Despite the discrepancies in the reproduction of the incident wave, these results are a vast improvement on those predicted by both linear and second-order diffraction theory when applied to steep nonlinear regular wave interactions with a bottom mounted cylinder [44.

\section{Hemispherical-bottomed Buoy with Linear Mooring}

Increasing the complexity of the model, a floating, hemispherical-bottomed, 440 cylindrical buoy with a linearly-elastic mooring has been simulated using the NWT. The buoy is part of the SuperGen UK Centre for Marine Energy Research (UKCMER) grand challenge project X-MED eXtreme loading of Marine Energy Devices due to waves, currents, flotsam and mammal impact which aims to identify and improve the understanding of extreme loading on tidal stream 

is $0.5 \mathrm{~m}$ in diameter with a $0.25 \mathrm{~m}$ tall cylindrical section above its hemispherical bottom (Figure 7). It is constructed from $2 \mathrm{~mm}$ thick mild steel and has ballast weight secured within the hemispherical part. The total mass is $43.2 \mathrm{~kg}$ centred $0.181 \mathrm{~m}$ above the bottom mooring fixing. The moment of inertia of the buoy is (1.61 1.610 .5$) \mathrm{N}$ m. Restrained only by a single-point mooring, which attaches at the bottom of the buoy where the symmetry axis intersects the surface of the hemisphere, the structure in this case is able to move in all six degrees of freedom (6DOF). The mooring has a stiffness of $67 \mathrm{~N} \mathrm{~m}^{-1}$ and a rest length of $2.18 \mathrm{~m}$
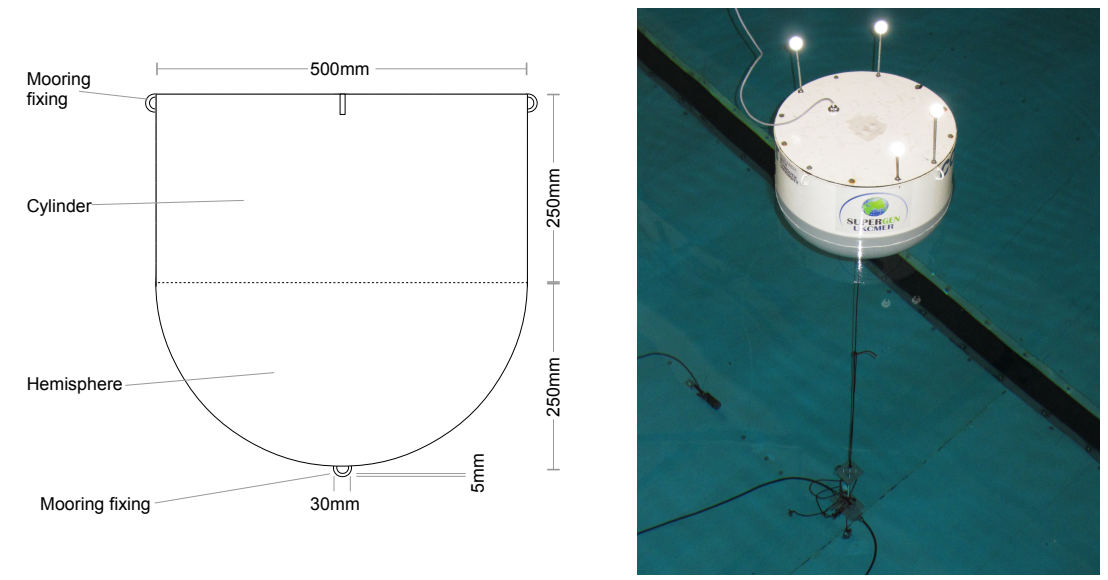

Figure 7: Dimensions of the hemispherical-bottomed, cylindrical buoy (left), and; a photograph from the COAST laboratory (right) showing the single-point, linear mooring and experimental setup.

\subsection{Background - 6DOF rigid body motion}

In terms of similar research in the literature, full $6 \mathrm{DOF}$ motion is rarely considered when simplified dynamics can be assumed instead. For the majority of moored WECs, however, this is not an option as the behaviour of the body, due to wave excitation, is highly dependent on multiple coupled modes of motion. 
For example, in their summary of the water entry of a wedge using fully nonlinear potential theory, $\mathrm{Wu}$ et al. [50] showed that asymmetry, horizontal velocity and rotational velocity all have significant effects on the flow and pressure and that there are strong couplings between these three degrees of freedom. Bai and Eatock-Taylor [51] modelled the fully nonlinear radiated waves resulting from the forced oscillation of a truncated cylinder using a high order boundary element method. They found their model to be accurate, efficient and stable. However, of most interest was their discovery that strong nonlinear interactions exist between the heave and pitch motions. These cause a total run-up, in the combined case, that differs significantly from the linear superposition produced by the two motions independently.

The majority of nonlinear time-domain investigations into the motion of fully-coupled freely-floating bodies and mooring restraints are concerned with vessels like Floating Production Storage and Offloadings (FPSOs) and Liquid Natural Gas (LNG) carriers. Xing et al. 52 obtained very good agreement between results from their Navier-Stokes (NS) solver and physical experiments of a 2D free-floating rectangular body in small regular waves. Yang et al. [53. used a NS solver with a $\mathrm{VOF}$ treatment for the free surface to model successfully nonlinear wave interactions, green water and the motion of a freely-floating LNG carrier but provided no physical validation of their results. Lu [54] modelled an FPSO able to move in heave and pitch in extreme regular waves using a NS solver and VOF method but found a number of differences in both the calculated motion and deck water height when compared to equivalent physical measurements. Despite this, Lu $[54$ went on to investigate the effect of various mooring arrangements on both a single FPSO and for a pair of vessels. A linearly elastic mooring model was used with the compression force set to zero. It was found that mooring restraints could be used to reduce the green water loads on the vessel but no validation for the moored cases was offered. Zhao et al. [55] investigated the interactions between extreme waves and a floating body able „o move freely in both heave and roll. Their NS solver, with the Constrained ${ }_{490}$ Interpolation Profile (CIP) scheme based on a Cartesian grid method, gave good 
comparisons with physical results for green water, impact pressures and motion response in regular, focused and combined waves. However, their model was not able to reproduce the peak pressure from a secondary loading cycle observed in the focused and combined wave experiments. Furthermore, their model tended

where $F_{\text {restraint }}$ is the force exerted in the direction of a vector from the mooring fixing on the buoy to the anchor position on the basin floor, $k_{\text {spring }}$ is the spring constant or stiffness in $\mathrm{N} \mathrm{m}^{-1}$ and $e$ is the extension of the spring from its rest length. Clearly in Equation 4 a negative $e$ (compression) is possible, producing a force that repels the buoy, however, this would not be the case in reality as 
for a mooring with mass, the direction and magnitude of the restraint force will be altered by the sag in the line and the dynamics of its motion. These two effects have not been considered here.

\subsection{Results - Buoy}

520 an initial test was conducted in which the buoy was released from an elevated position above still water and its heave displacement allowed to decay over time. This 'decay' test was performed with, and without, the mooring present in order to evaluate the accuracy of the motion prediction and the stability of the solver. buoy was positioned at the target location of NewWave case 2 above.

\subsubsection{Decay tests}

For the decay tests, the buoy was located, in an elevated position, in the centre of the domain, which had a square cross-section in the horizontal plane 530 (Figure 8). The width of the domain was $4 \mathrm{~m}$. The water depth was $2.8 \mathrm{~m}$ and the height of the air phase was $1.5 \mathrm{~m}$. The mesh was constructed from cubic cells with a background mesh cell side-length of $0.17 \mathrm{~m}$. The region containing the free-surface was refined to a level of 3 and the mesh close to the buoy was refined to level 4 (Figure 8 8 ). A cylindrical relaxation zone with an inner radius of $1 \mathrm{~m}$ and an outer radius of $2.83 \mathrm{~m}$ was centred on the buoy in order to absorb any radiated waves that might affect the results (Figure 8b).

The results from the decay tests are shown in Figure 9. It can be seen that the $6 \mathrm{DOF}$ solver in OpenFOAM ${ }^{\circledR}$ performs very well, particularly in the case with the mooring attached (Figure 9b). The unmoored case (Figure 9a) displays a slight inaccuracy in the frequency of oscillation and towards the end of the time series the solutions diverge from one another. This may be evidence of an issue with the force calculation or the pressure boundary condition on the surface of the buoy, however, this behaviour was not seen in the moored case. This may 

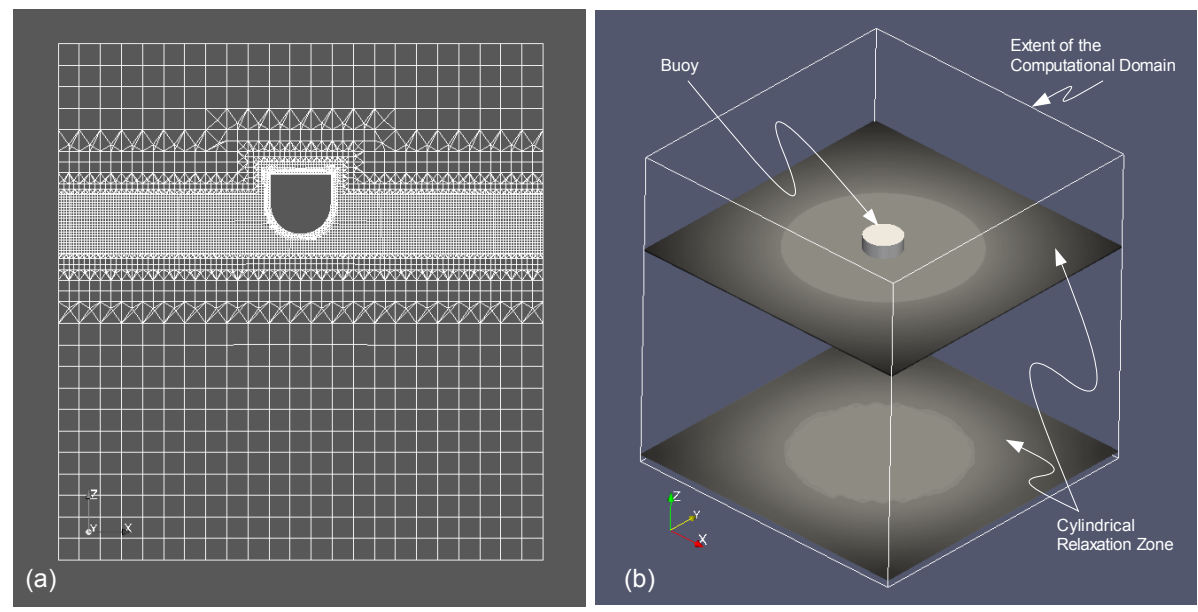

Figure 8: The computational domain set-up for the moored buoy decay tests. (a) a crosssection of the mesh; (b) the domain extents and cylindrical relaxation zone.

be a consequence of the additional driving force in the mooring dominating the motion and perhaps restricting additional degrees of freedom from becoming active. It is possible that the 'direction' of the interpolation scheme used could cause a slight asymmetry in the forcing which could excite other modes of motion but this does not seem to be the case here. It was found that the results were highly sensitive to the domain size and the amount of wave absorption, i.e. the influence of radiated waves reflecting from the domain boundaries, but no improvement on these results could be achieved by increasing the domain size or altering the relaxation zone. A common concern with the dynamic mesh treatment in OpenFOAM ${ }^{\circledR}$ is that large motions cause the mesh to degrade and it has been speculated that artificial mesh 'stiffness' can result from this mesh deformation [26]. In the case of this decay test, the buoy is released with the mesh undeformed and oscillates about its equilibrium state, which has a deformed mesh. This may cause issues that would not otherwise be a problem when the buoy begins in its equilibrium state. However, if this deformation were to be an issue, one might expect the associated error to increase with the displacement of the buoy, but of the two cases in Figure 9, the moored case has 

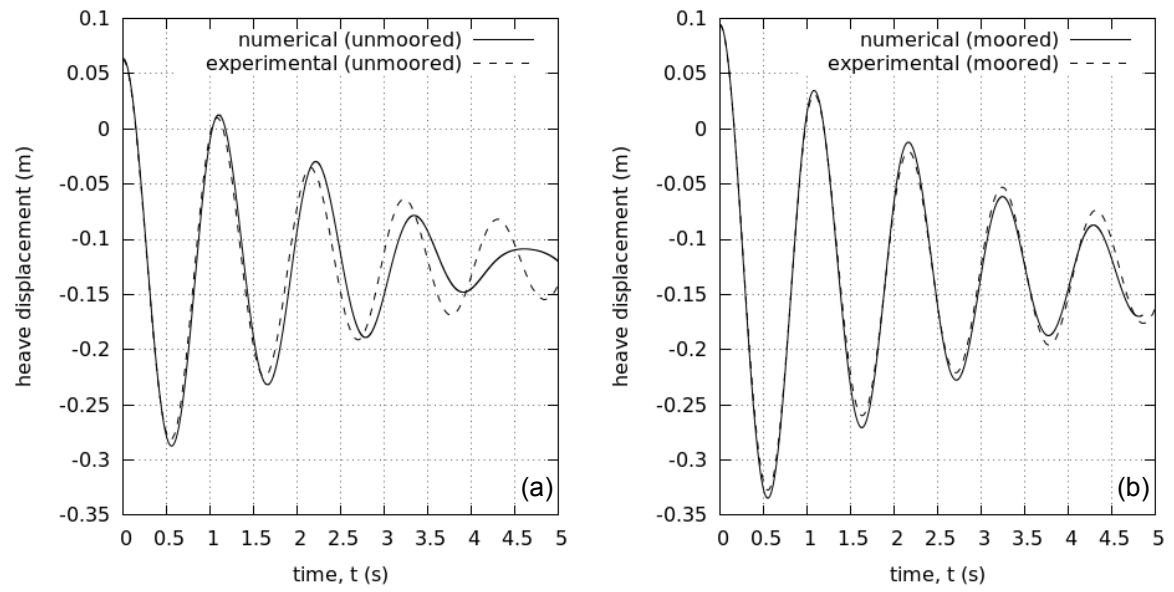

Figure 9: Comparisons between the heave displacement of a buoy in a physical (grey) and equivalent numerical (black) decay test with (right) and without (left) the mooring attached

a greater displacement and a better result.

\subsubsection{NewWave test}

In the focused wave case, the buoy was positioned $5.49 \mathrm{~m}$ from the inlet and subject to same 100 year wave event as in the NewWave case 2 above. The computational domain was $18 \mathrm{~m}$ long and had a width of $6 \mathrm{~m}$. The mesh resolution was kept the same as in the wave-only case but expanded to have cubic cells. In addition, a cylindrical region of the mesh, with a radius of $2 \mathrm{~m}$ and a depth of $1.5 \mathrm{~m}$, centred on the buoy was refined one level and the region in close proximity to the buoy was refined two levels.

Figure 10 shows a series of vertical cross-sectional snapshots from the simulation at a number of times either side of the arrival of the main crest in the wave packet. The mesh design and the modest amount of mesh deformation caused by the buoy's motion can be seen as well as the free surface across $\approx 10 \mathrm{~m}$ of the NWT. Clearly the buoy is displaced by the wave in heave, surge and pitch. The mooring has not been shown in Figure 10 but its effect has been included in the simulation. 

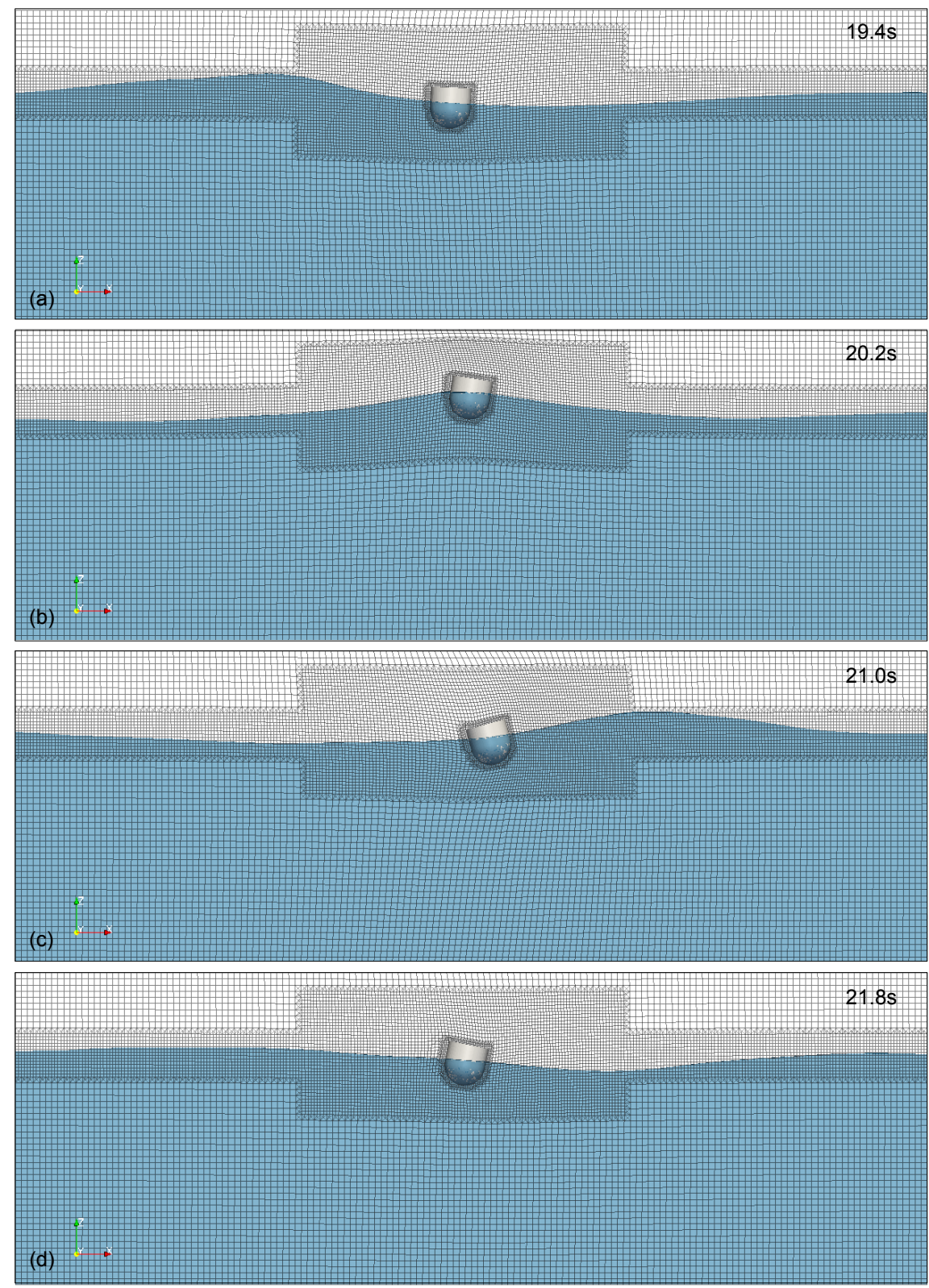

Figure 10: A series of vertical cross-sectional snapshots of a moored buoy simulation showing the mesh and free surface at times either side of the arrival of an extreme wave. 

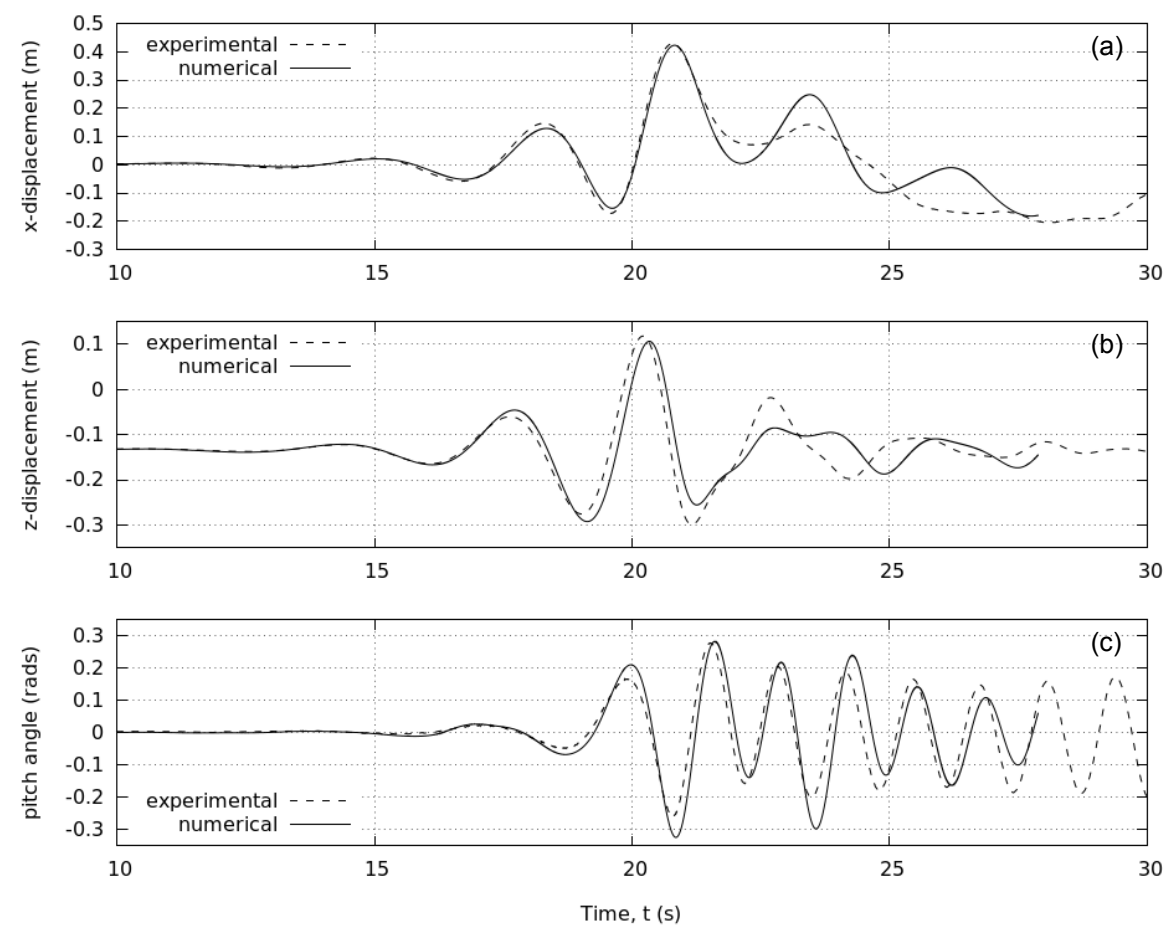

Figure 11: Numerical and experimental results for (a) the x-displacement, (b) z-displacement and (c) pitch angle of a buoy subject to an extreme wave event.

For a more quantitative analysis of the buoy's motion response, Figure 11 shows the resulting time series for the horizontal displacement of the centre of mass in the direction of wave propagation (Figure 11a), the vertical displacement (Figure 11b) and the pitch angle of the buoy (Figure 11:) compared with measurements taken in the physical experiment using the Qualysis motion capture system [15]. As can be seen, the agreement is very good until after the main wave crest has passed, when the discrepancies are believed to be due to reflections from the end and side walls of the NWT. The motion of the buoy has been captured very well; the phase of the motion response matches that of the physical buoy, apart from a slight delay in the heave motion (Figure 11?), and both the peak heave and surge displacements are within a few percent of 


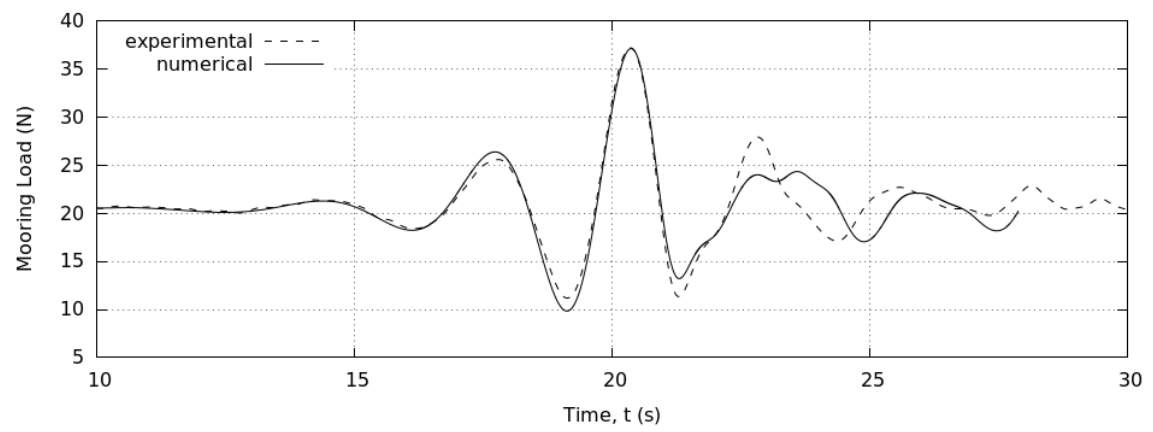

Figure 12: Numerical and experimental results for the load in the mooring of a buoy subject to an extreme wave event

the physical values. The amplitude of the pitch motion is less accurate on some of the oscillations but in comparison with similar work in the literature [54, 56, this is a very good reproduction of the buoy's motion. After the passing of the main wave crest, reflections begin to affect the numerical result due to the NWT being relatively short and narrow in order to reduce CPU effort. The very good agreement between the numerical and physical results is a clear consequence of a high quality reproduction of the wave only case (Figure 2), but also evidence that the $6 \mathrm{DOF}$ rigid-body solver performs very well even for reasonably large displacements.

Finally, the load in the mooring, prescribed by Equation 4 and based on the position of the float in the numerical simulation, is shown in Figure 12 along with the measurements made during the physical experiment. Again the agreement is very good until after the main crest where reflections have altered the motion of the buoy in the numerical case. Although the troughs either side show some differences, the peak load has been reproduced to within a remarkakble $0.2 \%$ of the physical result.

The $28 \mathrm{~s}$ simulation took almost 500 hours of CPU time running on 6 Intel ${ }^{\circledR}$ Xeon(R) CPU E5630 @ $2.53 \mathrm{GHz}$ processors. This is excessive and part of the reason why full $\mathrm{NS}$ solvers are seen as unwieldy for use in device design. It is possible that the execution time can be greatly reduced by decomposing 
the domain across many more processors, and recent investigations using the ARCHER UK National High Powered Computing (HPC) Facility and large numbers of processors have shown a dramatic reducing in the execution time of this case $(\approx 10$-fold increase in speed $)$.

\section{Conclusions}

The computational tool developed here is capable of reproducing a design wave based on the 100 year event at the Wave Hub site. The reproduction does however appear to be particularly sensitive to the quality of the prescribed wavemaker boundary conditions and there is still some concern over the use of linear superposition to generate highly nonlinear waves, particularly in shallower water depths. Improved boundary definitions of the full range of flow variables will undoubted improve the reproduction of extreme waves as well as applications involving such events i.e. survivability testing of WECs

Despite this, the NWT described in this work has been shown to accurately predict the run-up and pressure on the front face of a generic WEC hull geometry when fixed and subject to the 100 year wave event at the Wave Hub site. Furthermore, highly nonlinear phenomena including the secondary loading cycle, synonymous with the ringing of a vertical cylinder in steep non-breaking waves, and the characteristic free-surface behaviour in the vicinity of the cylinder have been reproduced well.

With the extension to $6 \mathrm{DOF}$ motion, the NWT performs very well. For the interaction between the 100 year wave event and a simplified WEC, consisting so a free-floating buoy and linear mooring, the motion of the buoy and load in the mooring has been reproduced remarkably well. It is clear that the NWT needs to be carefully designed for each case to ensure potential reflections from the side walls of the computational domain do not effect the results. However, in the case of focused waves these reflections typically only affect the results after the point of interest (the focus event).

In conclusion, the numerical tool presented here is able to provide a reliable 
reproduction of the fully nonlinear fluid dynamics associated with the interaction of extreme waves and WECs. This includes: the propagation of design waves, the free-surface behaviour around structures, the pressure on the structure's surface, the motion of floating structures and the loading in mooring lines. All of which are crucial in the assessment of WEC survivability. Therefore, the authors believe that numerical models, similar to the one presented here, can and should be used in the routine design of WECs in order to complement physical survival testing.

For guidelines and a more detailed discussion of the difficulties associated with reliable simulations of wave structure interaction in OpenFOAM ${ }^{\circledR}$, please refer to Ransley [20].

\section{Acknowledgements}

The authors would like to acknowledge the COAST Laboratory technical staff for their assistance in designing and running the physical experiments. The numerical work has been funded by the Engineering and Physical Sciences Research Council (EPSRC) via the SuperGen UK Centre for Marine Energy Research (UKCMER) as part of the third phase of the consortium's program of work (October 2011 - September 2016).

\section{References}

[1] B. Drew, A. R. Plummer, M. N. Sahinkaya, A review of wave energy converter technology, in: Proceedings of the Institution of Mechanical Engineers, Part A: Journal of Power and Energy, vol. 223, no. 8, 2009.

[2] A. F. O. Falcão, Wave energy utilization: A review of the technologies, Renewable and Sustainable Energy Reviews 14 (2010) 899-918.

[3] The Carbon Trust, Guidelines on design and operation of wave energy converters, 2005. 
[4] UK Energy Research Centre, UKERC Marine (Wave and Tidal Current) Renewable Energy Technology Roadmap - Summary Report, Tech. Rep., University of Edinburgh, 2008.

[5] EMEC, Guidelines for Reliability, Maintainability and Survivability of Marine Energy Conversion Systems, The European Marine Energy Centre Ltd, 2009 .

[6] S. Haver, O. J. Anderson, Freak Waves: Rare Realization of a Typical Population or Typical Realization of a Rare Population?, in: Proceedings of the 10th International Offshore and Polar Engineering Conference (ISOPE), Seattle, USA., 2000.

[7] D. Faulkner, Rogue Waves - Defining their Characteristics for Marine Design, in: Rogue Waves 2000, Brest, France., 2000.

${ }_{675}^{6}$ [8] P. R. Thies, J. Flinn, G. H. Smith, Reliability assessment and criticality analysis for Wave Energy Converters, in: Proceedings of the 8th European Wave and Tidal Energy Conference, Uppsala, Sweden, 2009.

[9] R. W. Yemm, R. M. Henderson, C. A. E. Taylor, The OPD Pelamis WEC: Current Status and Onward Programme., Ocean Power Delivery Ltd., Edinburgh, UK., 2000.

[10] EMEC, Tank Testing of Wave Energy Conversion Systems, The European Marine Energy Centre Ltd, 2009.

[11] P. S. Tromans, A. R. Anaturk, P. Hagemeijer, A New Model for the Kinematics of Large Ocean Waves - Application as a Design Wave, in: Proceedings of the 1st International Offshore and Polar Engineering Conference, Edinburgh, UK, 64-71, 1991.

[12] D. A. G. Walker, P. H. Taylor, R. Eatock Taylor, The shape of large surface waves on the open sea and the Draupner New Year wave, Applied Ocean Research 26 (3) (2005) 73-83. 
[13] W. J. D. Bateman, V. Katsardi, C. Swan, Extreme ocean waves. Part 1. The practical application of fully nonlinear wave modelling, Applied Ocean Research 34 (2012) 209-224.

[14] H. Santo, P. H. Taylor, R. Eatock Taylor, Y. S. Choo, Average Properties of the Largest Waves in Hurricane Camille, Journal of Offshore Mechanics and Arctic Engineering 135.

[15] M. Hann, D. Greaves, A. Raby, Snatch loading of a single taut moored floating wave energy converter due to focussed wave groups, Ocean Engineering 96 (2015) 258-271.

[16] SuperGen Marine, SuperGen Marine energy Research - Full Report, Tech. Rep., University of Edinburgh, 2011.

[17] J. Cruz, Ocean Wave Energy. Current Status and Furture Perspectives, Springer, 2008.

[18] BMT Fluid Mechanics Ltd, Review of model testing requirements for FPSO's, Health and Safety Executive, 2001.

[19] K. Ruehl, D. Bull, Wave Energy Development Roadmap: Design to commercialization, in: Harnessing the Power of the Ocean, proceedings of OCEANS '12, Hampton Road, VA, 1-10, 2012.

[20] E. Ransley, Survivability of Wave Energy Converter and Mooring Coupled System using CFD, Ph.D. thesis, Plymouth University, 2015.

[21] H. Jasak, Error Analysis and Estimation for the Finite Volume Method with Applications to Fluid Flows, Ph.D. thesis, Imperial College of Science, Technology and Medicine, 1996.

[22] H. Rusche, Computational fluid dynamics of dispersed two-phase flows at high phase fractions, Ph.D. thesis, Imperial College of Science, Technology \& Medicine, 2002. 
[23] P. Higuera, J. L. Lara, I. J. Losada, Realistic wave generation and active wave absorption for Navier-Stokes models Aplication to OpenFOAM ${ }^{\circledR}$, Coastal Engineering 71 (2013) 102-118.

[24] N. G. Jacobsen, D. R. Fuhrman, J. Fredsøe, A wave generation toolbox for the open-source CFD library: OpenFOAM ${ }^{\circledR}$, International Journal for Numerical Methods in Fluids 70 (2012) 1073-1088.

[25] N. G. Jacobsen, Contrib/waves2Foam, website, http://openfoamwiki. net/index.php/Contrib/waves2Foam accessed 15th September 2014, 2014.

[26] OpenCFD, OpenFOAM ${ }^{\circledR}$, website, http://www.openfoam.org/ accessed 29th May 2014, 2014.

[27] G. F. Clauss, Freak Waves and Their Interaction with Ships and Offshore Structures, in: Q. Ma (Ed.), Advances in Numerical Simulation of Nonlinear Water Waves, World Scientific Publishing Co. Pte. Ltd, 641-690, 2010.

[28] S. R. Massel, Ocean Surface Waves - Their Physics and Prediction, vol. 36 of Advanced Series on Ocean Engineering, World Scientific, 2013.

[29] J. B. Rozario, P. S. Tromans, P. H. Taylor, M. Efthymiou, Comparison of Loads Predicted Using "NewWave" and Other Wave Models with Measurements on the Tern Structure, in: Wave Kinematics and Environmental Forces, vol. 29 of Advances in Underwater Technology, Ocean Science and Offshore Engineering, Springer Netherlands, 143-159, 1993.

[30] A. C. Hunt-Raby, A. G. L. Borthwick, P. K. Stansby, R. H. Taylor, Experimental measurements of focused wave group and solitary wave overtopping, Journal of Hydraulic Research 49 (4) (2011) 450-464.

[31] P. H. Taylor, B. A. Williams, Wave Statistics for Intermediate Depth Water - NewWaves and Symmetry, Journal of Offshore Mechanics and Artic Engineering 126 (55) (2004) 629-634. 
[32] P. Jonathon, P. H. Taylor, On Irregular, Nonlinear Waves in a Spread Sea, Journal of Offshore Mechanics and Artic Engineering 119 (37).

[33] S. F. Smith, C. Swan, Extreme two-dimensional water waves: an assessment of potential design solutions, Ocean Engineering 29 (2002) 387-416.

[34] C. N. Whittaker, A. Raby, C. J. Fitzgerald, P. H. Taylor, The average shape of large waves in the coastal zone, Coastal Engineering 114 (2016) $252-264$.

[35] H. Bredmose, N. G. Jacobsen, Breaking wave impacts on offshore wind turbine foundations: Focused wave groups and CFD, in: Proceedings of the ASME 29th International Conference on Ocean, Offshore and Arctic Engineering (OMAE), Shanghai, China, 2010.

[36] L. F. Chen, J. Zang, A. J. Hillis, G. C. J. Morgan, A. R. Plummer, Numerical investigation of wave-structure interaction using OpenFOAM, Ocean Engineering 88 (2014) 91-109.

[37] A. Hunt, Extreme Waves, Overtopping and Flooding at Sea Defences, Ph.D. thesis, Oxford University, 2003.

[38] E. Ransley, M. Hann, D. Greaves, A. Raby, D. Simmonds, Numerical and physical modeling of extreme waves at Wave Hub, Journal of Coastal Research Special Issue No. 65 (2013) 1645-1650.

[39] Wave Hub Ltd., Wave Hub, website, http://www.wavehub.co.uk/ accessed 9th January 2015, 2015.

[40] C. H. Kim, Y. Xu, J. Zou, Impact And Nonimpact On Vertical Truncated Cylinder Due to Strong And Weak Asymmetric Wave, International Journal of Offshore and Polar Engineering 7 (3) (1997) 161-167.

[41] J. Morison, M. P. O'Brien, J. W. Johnson, S. A. Schaaf, The forces exerted by surface waves on piles, Journal of Petroleum Technology 2 (5) (1950) 149-154. 
[42] H. Cao, J. Zha, D. Wan, Numerical simulation of wave run-up around a vertical cylinder, in: Proceedings of the 21st International Offshore and Polar Engineering Conference, Maui, Hawaii, USA, 2011.

[43] P. Ferrant, Š. Malencia, B. Molin, Nonlinear wave loads and runup on a vertical cylinder, in: O. Mahrenholtz, M. Markiewicz (Eds.), Nonlinear Water Wave Interaction, WIT Press, 252, 1999.

[44] D. L. Kriebel, Nonlinear wave interaction with a vertical circular cylinder. Part II: Wave run-up, Ocean Engineering 19 (1) (1992) 75-99.

[45] K. Irschik, U. Sparboom, H. Oumeraci, Breaking wave loads on a slender pile in shallow water, in: Proceedings of the 29th International Conferencein Coastal Engineering (ICCE), Lisbon, 2004.

[46] J. Wienke, H. Oumeraci, Breaking wave impact force on a vertical and inclined slender pile - theoretical and large-scale model investigations, Coastal Engineering 52 (2005) 435-462.

${ }_{785}$ [47] H. Bredmose, J. Skourup, E. A. Hansen, E. D. Christensen, L. M. Pedersen, A. Mitzlaff, Numerical reproduction of extreme wave loads on a gravity wind turbine foundation, in: Proceedings of the 25th International Conference on Offshore Mechanics and Arctic Engineering (OMAE), Hamburg, Germany, 2006.

[48] J. R. Chaplin, R. C. T. Rainey, R. W. Yemm, Ringing of a vertical cylinder in waves, Journal of Fluid Mechanics 350 (1997) 119-147.

[49] SuperGen UKCMER, SuperGen UK Centre for Marine Energy Research (UKCMER) website, online, http://www.supergen-marine.org. uk/drupal/ accessed 8th February 2015, 2015.

${ }_{795}$ [50] G. X. Wu, G. D. Xu, W. Y. Duan, A summary of water entry problem of a wedge based on the fully nonlinear velocity potential theory, in: The Proceedings of the 9th International Conference on Hydrodynamics, Shanghai, China, 2010. 
[51] W. Bai, R. Eatock-Taylor, Higher-order boundary element simulation of fully nonlinear wave radiation by oscillating vertical cylinders, Applied Ocean Research 28 (2006) 247-265.

[52] Y. Xing, I. Hadzic, S. Muzaferija, M. Peric, CFD Simulation of Flowinduced Floating-body Motions, in: The Proceedings of the 16th International Workshop on Water Waves and Floating Bodies, Hiroshima, Japan, 2001.

[53] C. Yang, R. Löhner, H. Lu, An unstructured-grid based volume-of-fluid method for extreme wave and freely-floating structure interactions, Journal of Hydrodynamics, Ser. B 18 (3) (2006) 415-422.

[54] H. Lu, On the simulation of ship motions induced by extreme waves, Ph.D. thesis, George Mason University, 2009.

[55] X. Zhao, C. Hu, Z. Sun, Numerical simulation of extreme wave generation using VOF method, Journal of Hydrodynamics 22 (4) (2010) 466-477.

[56] J. Westphalen, Extreme Wave Loading on Offshore Wave Energy Devices using CFD, Ph.D. thesis, Plymouth University, 2010. 